\title{
1 PREVALENCE OF UgT1A1 GeNETIC VARIANTS IN ARGENTINEAN POPULATION, \\ 2 POTENTIAL IMPLICATIONS FOR PHARMACOGENOMIC TESTING
}

3

4 Cintia V. Cruz ${ }^{1,2}$, Paula Scibona1, Waldo H. Belloso¹.

5

1. Clinical Pharmacology Section, Internal Medicine Service. Hospital Italiano de Buenos Aires Juan Domingo Perón 4190 (CP C1181) Ciudad Autónoma de Buenos Aires, Argentina. Universidad de Buenos Aires

Paraguay 2155 (CP C1121)

\author{
Ciudad Autónoma de Buenos Aires, Argentina
}

\section{ABSTRACT} relevant for pharmacogenetic research. intestinal, stomach or breast tissue.

24 for UGT1 isozymes for a population of 100 healthy argentinians.

2. Laboratorio de Estadística Aplicada a las Ciencias de la Salud (LEACS) Departamento de Farmacología y Toxicología 2da Unidad Académica

The four groups of uridine diphosphate glucuronosyltransferase (UGT) enzymes form a superfamily responsible for the glucuronidation of target substrates. These include hormones, flavonoids, environmental mutagens and pharmaceutical drugs. Thus, UGT enzymes are

Most of the members of the UGT family are expressed in the liver, but are also present in

The incidences and types of polymorphisms for different enzymes vary with geographical regions and ethnic groups. This is the first study that examined the frequency of polymorphisms

25 The distribution of UGT1A1 in our population was: $70.5 \%(70.5)$ for the *1 allele, $21.5 \%$ for the $6{ }^{*} 28$ allele and $1 \%$ for the *36 allele. $48 \%$ (48) presented the * $1 /{ }^{*} 1$ genotype, while $43 \%$ (43) had 
(7) showed ${ }^{*} 28 /{ }^{*} 28$. There was no preferential sex

28 distribution.

29 Since most Argentinians are of Caucasian descent, a European genotype frequency profile is to

30 be expected. That is evident in the wild type prevalence in our population. However, the

31 contribution of Native American ancestry to gene pool components may in part explain the

32 higher prevalence of the *28 genotype in UGT1A1 *1 in our population, in comparison with

\section{European cohorts.}

\section{INTRODUCTION}

35 The uridine diphosphate glucuronosyltransferase (UGT) enzymes conform a superfamily of enzymes responsible for the glucuronidation of target substrates. The transfer of glucuronic acid

37 renders xenobiotics and other endogenous compounds water soluble, allowing for their biliary or renal excretion. The UGT superfamily is responsible for the glucuronidation of hundreds of compounds, including hormones, flavonoids and environmental mutagens. Most of the members of the UGT family are expressed in the liver, as well as in other type of tissues, such

41 as intestinal, stomach or breast. A few members are expressed only outside the liver such as

42 UGT1A7, UGT1A8, UGT1A10 and UGT2A1. UGT superfamily is composed by four families:

43 UGT1A, UGT2, UGT3 and UGT8. UGT2 is further divided into two subfamilies, UGT2A and

44 UGT2B, both of which present on chromosome 4. Although limited studies are already available

45 on UGT2A enzymes, they appear to be involved in the glucuronidation of compounds such as

46 phenolic odorants and polycyclic aromatic hydrocarbon metabolites. UGT2B proteins are mainly

47 responsible for the metabolism of steroids. The roles of UGT3 and UGT8 family members have

48 not been well characterized yet. 
49 The UGT1A family is located on chromosome $2 q 37(1)$, and members of this group

50 glucuronidate a large variety of compounds. Pharmaceutical drugs are also a common substrate

51 of the UGT family, turning the enzymes in this group relevant for pharmacogenetic research(2).

52 Bilirubin-UGT (UGT1A1) conjugates bilirubin with glucuronic acid, converting the bilirubin into a

53 water-soluble form that is readily excreted in bile. Mutations of bilirubin UDP-glucurunosyl

54 transferase causes hereditary unconjugated hyperbilirubinemias, including Crigler-Najjar and

55 Gilbert syndromes(3).

56 The genetic defect in patients with Gilbert syndrome involves the promoter region of

57 UGT1A1(4,5). Gilbert syndrome manifests only in people homozygous for the variant promoter.

58 As a result, its inheritance is most consistent with an autosomal recessive trait. However,

59 heterozygotes for the Gilbert genotype have higher average plasma bilirubin concentrations

60 compared with those with two wild-type alleles. It is estimated that 9 percent of individuals in the

61 Western general population world are homozygous for the variant promoter, and up to 42

62 percent are heterozygous(5).

63 Characterization of the UGT1A gene locus has permitted an understanding of the molecular

64 defects responsible for the Gilbert syndrome. The mutation responsible for Gilbert syndrome is

65 in the promoter region, upstream to exon 1 of UGT1A1. The normal sequence of the TATAA

66 element within the promoter is A[TA]6TAA. Caucasian and black patients with Gilbert syndrome

67 are homozygous for a longer version of the TATAA sequence, A[TA]7TAA, which causes

68 reduced production of bilirubin-UGT. This variant is termed UGT1A1*28(6).

69 This longer TATAA element has been found in all individuals with Gilbert syndrome studied in

70 the United States, Europe, and countries of the Middle East and South Asia. However, other

71 factors are probably involved in the expression of Gilbert phenotype since not all patients who

72 are homozygous for the variant promoter develop hyperbilirubinemia. Furthermore, in the 
73 Japanese population, other mutations within the coding regions of UGT1A1 can be the

74 underlying cause of the Gilbert phenotype.

75 Since bilirubin-UGT is involved in the glucuronidation of several important drugs, individuals with

76 Gilbert syndrome may be more susceptible to the toxic effect of substances that require

77 bilirubin-UGT-mediated hepatic glucuronidation prior to excretion. Gilbert syndrome is known to

78 increase the risk of drug toxicity with irinotecan and the hyperbilirubinemia associated with

79 atazanavir(2,7).

80 The active metabolite of irinotecan, SN-38, is glucuronized in the liver mainly by bilirubin-UGT.

81 The major dose-limiting toxicity of this drug is diarrhoea.In patients who inherit certain UGT1A1

82 polymorphisms, reduced glucuronidation of SN-38 leads to an increased incidence of diarrhoea.

83 The symptoms can be severe enough to warrant switching to other drugs. Thus, it is currently

84 recommended to test the UGT1A1 polymorphism prior to the administration of Irinotecan and to 85 adjust the dose according to the resulting genotype(8).

86 As previously stated, some drugs may induce hyperbilirubinemia in patients with Gilbert

87 syndrome. Atazanavir, an antiretroviral medication, is an inhibitor of bilirubin-UGT activity and is

88 associated with hyperbilirubinemia(9). Isolated hyperbilirubinemia has also been reported during

89 the treatment of hepatitis $C$ with peginterferon and ribavirin and in patients receiving pazopanib.

90 In such cases, discontinuation of therapy is usually not necessary(10).

91 The incidences and types of the polymorphisms for these enzymes are quite different according

92 to geographical regions and ethnic groups. The aim of this study is to estimate the prevalence of

93 UGT1A1 polymorphism in the Argentine population and to evaluate what clinical implications

94 this might have.

\section{MATERIAL AND METHODS}




\section{Study population}

98 One hundred random and anonymized DNA samples from healthy donors were analysed. The

99 Hospital Italiano de Buenos Aires DNA Bank collection project has the approval of the local

100 ethic committee and all the volunteer subjects signed an informed consent.

\section{Sample collection and DNA extraction}

102 Following an informed consent process, $10 \mathrm{~mL}$ of peripheral blood were collected from each 103 subject in 5-mL EDTA tubes. Whole blood samples were stored at $4^{\circ} \mathrm{C}$ until the time of 104 processing. Genomic DNA was extracted and purified using the QI Amp DNA Blood Mini kit 105 (QIAGEN).

\section{Genotyping of UGT1A1}

107 Genomic DNA was extracted from $200 \mu \mathrm{L}$ of whole blood using QIAamp DNA Mini kit (Qiagen, $108 \mathrm{GmbH}, \mathrm{D}-40724$ Hilden, Germany). The polymerase chain reaction (PCR) was performed in a 109 final volume of $20 \mu \mathrm{L}$. The forward primer was 5'- CAGCCTCAAGACCCCACA - 3' and the 110 reverse primer was 5'- TGCTCCTGCCAGAGGTTC -3'. The PCR conditions were 5 minutes at $11195^{\circ} \mathrm{C}$, followed by 35 cycles of 30 seconds at $95^{\circ} \mathrm{C}, 60$ seconds at $61^{\circ} \mathrm{C}, 60$ seconds at $72^{\circ} \mathrm{C}$, 112 and final extension for 10 minutes at $72^{\circ} \mathrm{C}$. The PCR product was detected on $2 \%$ agarose gels 113 by means of ethidium bromide staining. The presence of variant UGT1A1 was confirmed by 114 direct sequencing of PCR products on an automated ABI 3100 capillary sequencer (Applied 115 Biosystems, Foster City, Calif) using the Big Dye Terminator Cycle Sequencing Kit (Applied 116 Biosystems).

\section{STATISTICAL ANALYSIS}

118 Allelic and genotypic frequencies were expressed in absolute and percentage values in the 119 study population. Sample size calculation based on the estimation of a $4 \%$ prevalence of TT 120 genotype as published for european population was of 64 samples, while for a prevalence of 7 $121 \%$ as in global population was of 110 samples, with an alpha error of $5 \%$. The results obtained 122 were further evaluated for Hardy-Weinberg equilibrium. 


\section{RESULTS}

124 The genotyping of all samples was according to the method previously described. Among

125 the 100 subjects analysed, $49 \%$ (49) were male and $51 \%(51)$ were female with a median age 126 of 43 and a range of $32-80$ years.

127 In our population, the distribution of UGT1A1 was -Figure 1 attached separatedly-: 70.5\% 128 (70.5) for the * 1 allele, $21.5 \%$ for the ${ }^{*} 28$ allele and $1 \%$ for the ${ }^{*} 36$ allele. $48 \%$ (48) presented 129 the * $1 / * 1$ genotype, while $43 \%(43)$ had $* 1 / * 28,2 \%(2)$ had $* 1 /{ }^{*} 36$ and $7 \%(7)$ showed $* 28 /{ }^{*} 28$.

130 No preferential sex distribution was observed between genotypes.

\section{DISCUSSION}

132 UGT 1A1 is the major UGT isoform responsible for glucuronidation of bilirubin in human liver, 133 and it is capable of conjugating various phenols, anthraquinones and flavones. Mutations of 134 bilirubin UDP-glucurunosyl transferase causes hereditary unconjugated hyperbilirubinemia and can also alter the metabolism of certain drugs.

In our population of 100 healthy argentinian volunteers, the distribution of the UGT1A1 showed

137 a predominance of the *1 allele whereas the *28 allele showed a relatively high prevalence of $13821,5 \%$.

139 Compared to this, in a cohort of 245 healthy men and women, aged 20-40 years of Caucasians 140 and Asians the frequencies of the UGT1A1 genotypes were $53,7 \%$ for $6 / 6 * 1,34,8 \%$ for $6 / 7$ * 1 $14128,9,8 \%$ for $7 / 7{ }^{*} 28,0.8 \%$ for $5 / 6$ and $0.8 \%$ for $6 / 8 * 1-38$ promoter TA repeats. Both allele and 142 genotype frequencies varied by race $(P<0.02)$, with $11 \%$ of the Caucasians and none of the 143 Asians having the $7 / 7{ }^{*} 28$ genotype. Overall, $8 \%$ were homozygous variant for both UGT1 144 polymorphisms and $43 \%$ had at least one variant allele for both UGT1A1*28 and 145 UGT1A6*2(11).

146 The incidences and types of the polymorphisms for these enzymes vary according to 147 geographical regions and ethnic groups. This is the first study that has examined the frequency 148 of polymorphisms for UGT1 isozymes for a population of healthy argentinians. 
Since most Argentinian inhabitants are of Caucasian descent, a European genotype frequency profile is to be expected. That is evident in the wild type prevalence in our population and the one from Lampe et. al. However, the contribution of Native American ancestry to gene pool components may in part explain the higher prevalence of the *28 genotype in UGT1A1 *1 in our population, in comparison with the $11 \%$ observed in the european study. This finding provides additional information to the ongoing controversy regarding which population -if any- can be used as a general reference for Argentinian genotypic profiles.

156 A limitation of our results is that all the volunteers evaluated are from the Capital City of Buenos

157 Aires, Argentina. This does leave aside a considerable amount of variant genomics particularly

158 in a vast country that has a longstanding history of racial mixture.

159 Understanding these polymorphisms and the characterization of these in different populations is essential for the prevention of adverse effects of a considerable number of drugs and also for

161 the reduction of cancer-associated risks.

\section{BIBLIOGRAPHY}

163 1. Ritters JK, Crawford\$ JM, Owenssll IS. Cloning of Two Human Liver Bilirubin UDPcaused by genetic deficiency of UDP-glucuronosyltransferases. Toxicol Lett [Internet]. 2000 Mar 15 [cited 2019 Jul 25];112-113:333-40. Available from: http://www.ncbi.nlm.nih.gov/pubmed/10720749

3. Maruo Y, Iwai M, Mori A, Sato H, Takeuchi Y. Polymorphism of UDPGlucuronosyltransferase and Drug Metabolism. Curr Drug Metab [Internet]. 2005 Apr 1 [cited 2019 Aug 8];6(2):91-9. Available from: http://www.eurekaselect.com/openurl/content.php?genre=article\&issn=1389- 
4. Fretzayas A, Moustaki M, Liapi O, Karpathios T. Eponym. Eur J Pediatr [Internet]. 2012 Jan 9 [cited 2019 Jul 25];171(1):11-5. Available from: http://www.ncbi.nlm.nih.gov/pubmed/22160004

5. Bosma PJ, Chowdhury JR, Bakker C, Gantla S, de Boer A, Oostra BA, et al. The genetic basis of the reduced expression of bilirubin UDP-glucuronosyltransferase 1 in Gilbert's syndrome. N Engl J Med [Internet]. 1995 Nov 2 [cited 2019 Jul 25];333(18):1171-5. Available from: http://www.nejm.org/doi/abs/10.1056/NEJM199511023331802

6. Kaplan M, Hammerman C. Bilirubin and the Genome: The Hereditary Basis of Unconjugated Neonatal Hyperbilirubinemia [Internet]. Vol. 3, Current Pharmacogenomics. 2005 [cited 2019 Jul 25]. Available from: https://pdfs.semanticscholar.org/f2d5/9c4046254e0ec0d10b17ad2ad894ca41e1e4.pdf

7. Iyer L, King CD, Whitington PF, Green MD, Roy SK, Tephly TR, et al. Genetic

8. Table of Pharmacogenomic Biomarkers in Drug Labeling | FDA [Internet]. [cited 2019 Jul predisposition to the metabolism of irinotecan (CPT-11). Role of uridine diphosphate 25]. Available from: https://www.fda.gov/drugs/science-research-drugs/tablepharmacogenomic-biomarkers-drug-labeling glucuronosyltransferase isoform 1A1 in the glucuronidation of its active metabolite (SN38) in human liver microsomes. J Clin Invest [Internet]. 1998 Feb 15 [cited 2019 Jul 25];101(4):847-54. Available from: http://www.jci.org/articles/view/915

9. Laprise C, Baril J-G, Dufresne S, Trottier H. Atazanavir and Other Determinants of Hyperbilirubinemia in a Cohort of 1150 HIV-Positive Patients: Results from 9 Years of Follow-Up. AIDS Patient Care STDS [Internet]. 2013 Jul 5 [cited 2019 Jul 25];27(7):37886. Available from: http://www.liebertpub.com/doi/10.1089/apc.2013.0009

10. Zwirtes R, Narasimhan P, Wind-Rotolo MM, Xu D, Hruska MW, Kishnani N, et al. Mechanisms of Hyperbilirubinemia During Peginterferon Lambda-1a Therapy for Chronic 

Nov [cited 2019 Jul 25];36(11):644-51. Available from: http://www.ncbi.nlm.nih.gov/pubmed/27710263

204 11. Lampe JW, Bigler J, Horner NK, Potter JD. UDP-glucuronosyltransferase (UGT1A1*28 and UGT1A6*2) polymorphisms in Caucasians and Asians: relationships to serum bilirubin concentrations. Pharmacogenetics [Internet]. 1999 Jun [cited 2019 Jul 25];9(3):341-9. Available from: http://www.ncbi.nlm.nih.gov/pubmed/10471066 
Figure 1: UGT1A1 Genotypes distribution in Argentinian population

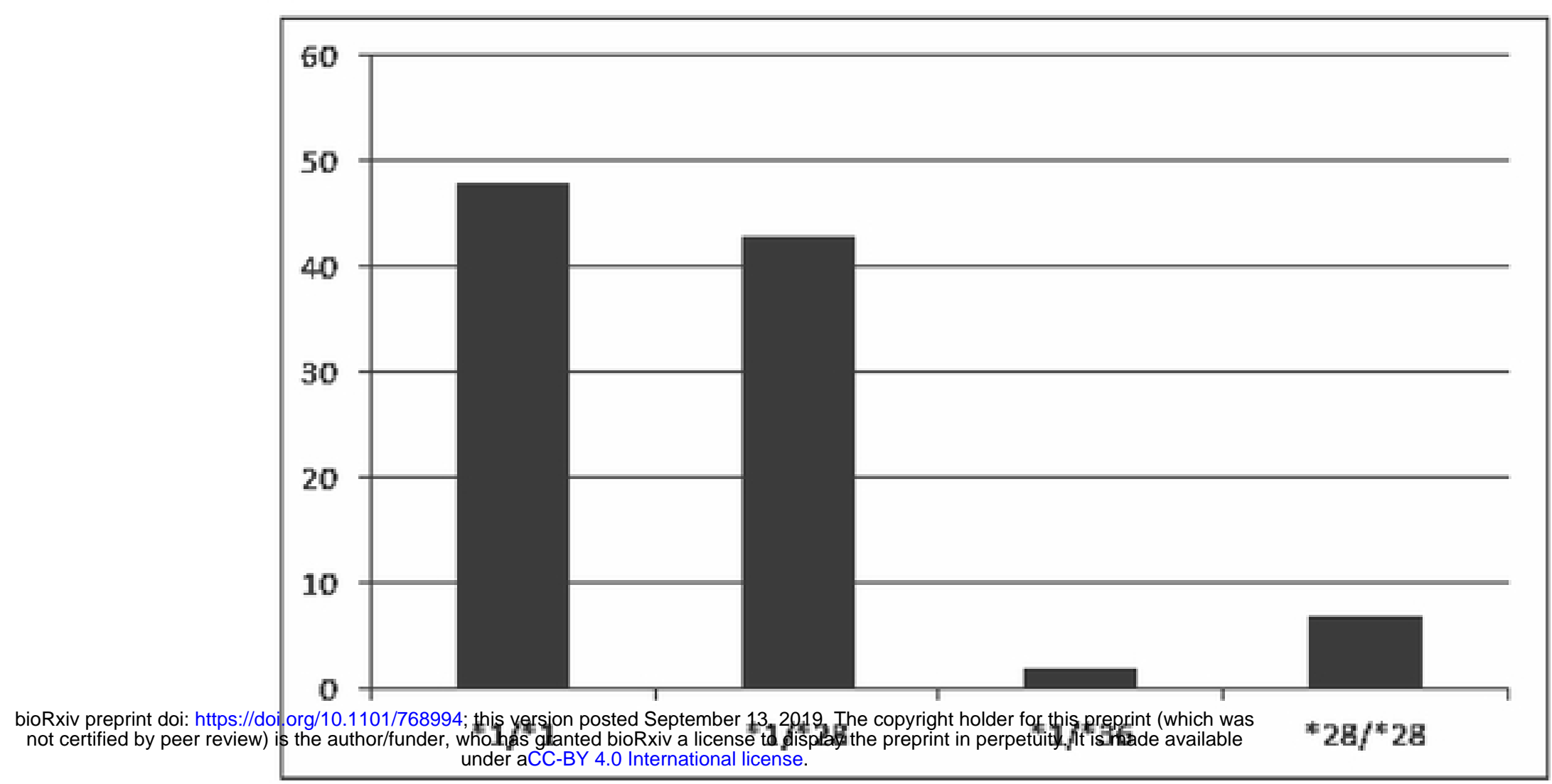

Figure 